\title{
CT scan chest findings in symptomatic COVID-19 patients: a reliable alternative for diagnosis
}

\author{
Benjamin D. Sarkodie and Yaw B. Mensah
}

Ghana Med J 2020; 54(4) supplement: 97-99 doi: http://dx.doi.org/10.4314/gmj.v54i4s.14

\author{
Department of Radiology, University of Ghana Medical School, Korle Bu, Accra
}

Corresponding author: Dr. Benjamin Dabo Sarkodie

E-mail: ghana_neo@yahoo.com

Conflict of interest: None declared

\section{SUMMARY}

Computed Tomography (CT) scan of the chest plays an important role in the diagnosis and management of Coronavirus disease 2019 (COVID-19), the disease caused by the novel coronavirus SARS-CoV-2. COVID-19 pneumonia shows typical CT Scan features which can aid diagnoses and therefore help in the early detection and isolation of infected patients. CT scanners are readily available in many parts of Ghana. It is able to show findings typical for COVID-19 infection of the chest, even in instances where Reverse Transcription Polymerase Chain Reaction (RTPCR) misses the diagnosis. Little is known about the diagnostic potential of chest CT scan and COVID-19 among physicians even though CT scan offers a high diagnostic accuracy.

Keywords: Computed Tomography, COVID-19, Coronavirus disease 2019, RT-PCR: Reverse Transcription Polymerase Chain Reaction

\section{INTRODUCTION}

The novel coronavirus disease 2019(COVID-19) is an infectious disease caused by the severe acute respiratory syndrome coronavirus2 (SARS-CoV-2). ${ }^{1}$ SARS-CoV-2 belongs to the coronavirus family responsible for respiratory complications.

The disease was reported in Wuhan, China, in December 2019 before spreading to many parts of the world by February, 2020. ${ }^{2}$ The World Health Organisation (WHO) confirmed reports of person-to-person spread and subsequently declared the disease a pandemic on $11^{\text {th }}$ March, 2020.3, COVID-19 has rapidly spread to over 210 countries and territories, with over 17.4 million cases and $675,584,000$ deaths reported globally as of August $31^{\text {st }}$, $2020{ }^{4}$ The Ghana Health Service as of $30^{\text {th }}$ July 2020, had documented 35,142 confirmed cases with 175 deaths in Ghana. ${ }^{5}$

Fever, cough and difficulty in breathing are some of the common COVID-19 symptoms. ${ }^{6,7}$ Like all epidemics, the key strategies to prevent widespread transmission is to test/diagnose, isolate and treat individuals with suspected or confirmed COVID-19.

\section{PCR testing issues and why CT scan of the chest}

The gold standard for testing for the novel coronavirus is the Reverse Transcription Polymerase Chain Reaction (RT-PCR) method. $8,9,10$
RT-PCR, or PCR swab has a specificity $99 \%$ with a sensitivity of $50-80 \%$ thus potentially up to $50 \%$ cases of COVID-19 could be missed by PCR.

Due to the highly transmissible nature of COVID-19, the number of individuals who require testing is typically very high and potentially overwhelming for healthcare system. Problems with testing efficiency, reliability and delays with results availability have previously been reported ${ }^{9}$ with obvious implications for disease control and transmission prevention. Test results in centres in Ghana have been received after $2-4$ weeks thus defeating the purpose of early diagnosis. In order to address this pitfall a lot of clinics in China at the peak of the COVID-19 infection did both PCR and chest CT for suspected cases. ${ }^{11}$

Studies in China showed that chest CT scan was able to pick some cases of COVID-19 pneumonia which were missed by the swab test. ${ }^{12}$ Many other studies have showed that CT scan of the chest is very reliable in the diagnoses of COVID-19 symptomatic patients. Several studies have reported that chest CT has a sensitivity of $80-90 \%$ and a specificity of $82.8-96 \%$ for detecting lung lesions in patients with COVID-19. ${ }^{13,14}$

The guidelines for Diagnosis and Treatment of Pneumonitis Caused by 2019-nCoV (trial sixth version) published by the Chinese Health Authority recommended chest CT as an effective method to screen suspicious cases. 
The addition of chest CT for diagnosis of COVID-19 resulted in several thousands of clinically diagnosed cases in China which played an important role in controlling the epidemic in China. ${ }^{15}$ A policy direction that will encourage the utilization of CT scan as a complementary or alternative tool in the diagnosis of COVID 19 will help consolidate national and global efforts at controlling the virus.

Beyond this sensitivity, CT also offers the advantage of being able to interpret findings immediately hence once COVID-19 infection is suspected, patients can be isolated and treated early to reduce risk of spreading the infection. As there is still no approved rapid swab testing in Ghana at the time of this study, relying solely on PCR testing can potentially slow progress to tackle this virus. We need to use CT scan of the chest as a reliable complement to PCR testing. Countries like China and Singapore that have achieved a significant level of control have deployed wide use of CT scan of the chest.

\section{CT Scan /PCR Discordance}

Between March and June 2020, we picked up 43 cases of COVID-19 chest infections on chest CT scans in a private clinic in Accra. Forty of these patients were subsequently confirmed by PCR during the first testing (40/43 Concordance). Of the remaining 3 , a second PCR testing done confirmed 2 of them as positive while the third patient remained negative. We insisted on retesting because the imaging findings were typical for COVID-19 according to previously published imaging findings ${ }^{16,17,18,19}$ including bilateral, peripheral and subpleural ground glass opacities. RT-PCR test for 2019-nCoV may be falsely negative due to laboratory error or insufficient viral material in the specimen. ${ }^{20}$

\section{Case presentation of the three cases who initially tested negative}

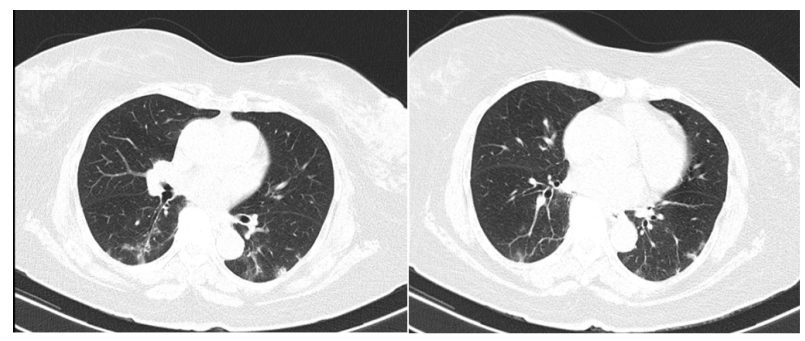

Case 1 Seventy-seven-year-old female with a 5-day history of cough with fever and headaches. CT scan of the chest showing bilateral subpleural mixed ground-glass opacities GGO and consolidation with traction bronchiectasis (Negative initial PCR testing. Positive repeat PCR within 3 days)

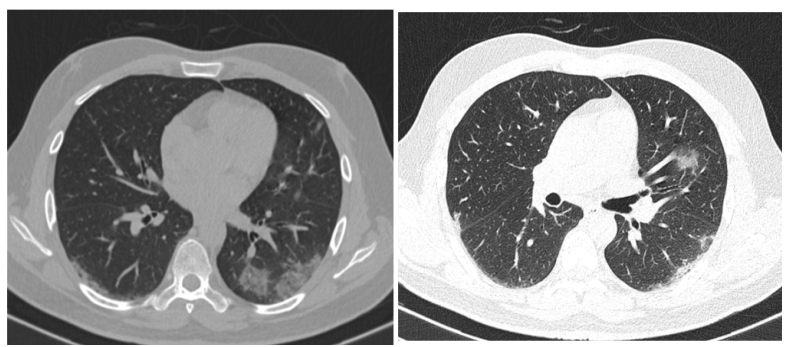

Case2 39-year-old male with history of vomiting and abdominal pain. No cough or fever. CT scan of the abdomen found incidental ground glass opacities at the lung bases for which a subsequent dedicated chest CT scan was requested showing bilateral and subpleural groundglass opacities (Negative initial PCR testing. Positive repeat PCR within 3 days)

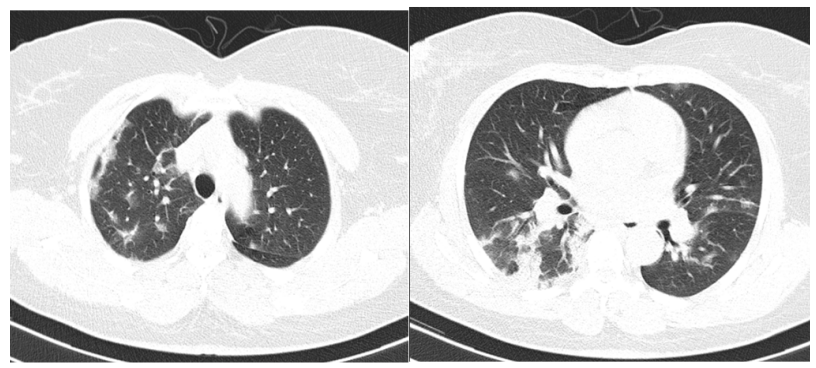

Case 3 50year old male with three-day history of cough and fever. CT scan of the chest showing bilateral, peripheral and subpleural consolidation (Negative initial PCR testing. Negative repeat PCR within 3 days)

In Ghana, a there are currently over $35 \mathrm{CT}$ scanners dotted across the country (according to a document obtained from the Ghana's Nuclear Regulatory Agency in 2008) compared to the only three centres with PCR testing capabilities. Using the CT scan for triaging COVID-19 cases is certainly worth considering in patients who are symptomatic.

There are disadvantages associated with the use of the CT scan for triaging COVID-19 patients like radiation exposure and the higher cost compared to PCR but the benefits of controlling the virus far out weights these downsides. Also, the current PCR laboratory testing is time-consuming, and often plagued by shortage of supply test kits and may not meet the needs of the growing infected population. RT-PCR testing for 2019-nCoV may be falsely negative due to laboratory error or insufficient viral material in the specimen. ${ }^{20}$

\section{CONCLUSION}

CT scan of the chest has been shown to have a high sensitivity and specificity for identifying lung lesions in COVID-19 infection. With the limited access to PCR testing and associated delay in receiving results the use 


\section{Special Article}

of CT scan of the chest in triaging should be encouraged. In China and Korea where the virus has been largely put under control there is a widespread of CT scan of the chest to aid in diagnosis.

\section{REFERENCES}

1. Shereen MA, Khan S, Kazmi A, Bashir N, et al. COVID-19 infection: Origin, transmission, and characteristics of human coronaviruses. Journal of Advanced Research.2020;24:91-98 doi.org/10.1016/j.jare.2020.03.005

2. Zhu, N., D. Zhang, W. Wang. China Novel Coronavirus Investigating and Research Team. A novel coronavirus from patients with pneumonia in China. $N$ Engl $J$ Med. 2020.382(8):727-3. doi: 10.1056/NEJMoa2001017

3. Bai Y, Yao L, Wei T, Tian F, et al. Presumed asymptomatic carrier transmission of COVID-19. JAMA 2020; 323(14): 1406-1407. doi:10.1001/jama.2020.2565

4. Johns Hopkins University and Medical Centre. Johns Hopkins Coronavirus Resource Center:Home[Internet]. 2020. Available from: https://coronavirus.jhu.edu/. Accessed 31 ${ }^{\text {st }}$ August, 2020.

5. Ghana Health Service. COVID-19 Updates | Ghana - Ghana Health Service[Internet]. 2020.Available from:https://www.ghanahealthservice.org/covid19/. Accessed $20^{\text {th }}$ July, 2020.

6. Yu P, Zhu J, Zhang Z, Han Y. A familial cluster of infection associated with the 2019 novel coronavirus indicating possible person-to-person transmission during the incubation period. The Journal of infectious diseases. 2020; 221(11):1757-1761. doi: 10.1093/infdis/jiaa077

7. Rabaan AA, Al-Ahmed SH, Haque S, Sah R, et al. SARS-CoV-2, SARS-CoV, and MERS-CoV: a comparative overview. Infez Med 2020; 28(2): 174184. PMID: 32275259

8. Yang $\mathrm{X}, \mathrm{Yu} \mathrm{Y}, \mathrm{Xu}$ J, Shu H, et al. Clinical course and outcomes of critically ill patients with SARSCoV-2 pneumonia in Wuhan, China: a single-centered, retrospective, observational study. The Lancet Respiratory Medicine. 2020 8(5): 475-481 doi: 10.1016/S2213-2600(20)30079-5

9. Lippi G, Simundic AM, Plebani M. Potential preanalytical and analytical vulnerabilities in the laboratory diagnosis of coronavirus disease 2019 (COVID19). Clinical Chemistry and Laboratory Medicine (CCLM). 2020;58(7):1070-1076.

doi: $10.1515 / \mathrm{cclm}-2020-0285$
10. Uhteg K, Jarrett J, Richards M, Howard C, et al. Comparing the analytical performance of three SARS-CoV-2 molecular diagnostic assays. Journal of Clinical Virology. 2020;127:104384 doi: 10.1016/j.jcv.2020.104384

11. Fang Y, Zhang H, Xie J, Lin M, et al. Sensitivity of chest CT for COVID-19: comparison to RTPCR. Radiology.2020; 296(2): $\quad$ E115-E117 doi.org/10.1148/radiol.2020200432

12. Xie X, Zhong Z, Zhao W, Zheng C, Wang F, Liu J. Chest CT for typical 2019-nCoV pneumonia: relationship to negative RT-PCR testing. Radiology.2020; 2020;296(2): E41-E45.doi: 10.1148/radiol.2020200343

13. Li K., Wu J., Wu F., Guo D., et al. The clinical and chest $\mathrm{CT}$ features associated with severe and critical COVID-19 pneumonia. Invest Radiol. 2020. 55(6):327-331DOI: 10.1097/rli.0000000000000672

14. Li L, Qin L, Xu Z, Yin Y, et al. Artificial intelligence distinguishes COVID-19 from community acquired pneumonia on chest CT. Radiology. 2020. 296(2):E65-E71. doi: 10.1148/radiol.2020200905.

15. Zu ZY, Jiang MD, Xu PP, Chen W, et al. Coronavirus disease 2019 (COVID-19): a perspective from China. Radiology. 2020; 296(2):E15-E25. doi: 10.1148/radiol.2020200490

16. Bernheim A, Mei X, Huang M, Yang Y, et al. Chest CT findings in coronavirus disease-19 (COVID-19): relationship to duration of infection. Radiology. 2020; 200463. 20 (2020), pp. 200463.doi.org/10.1148/radiol.2020200463

17. Lei P, Huang Z, Liu G, Wang P, Song W, et al. Clinical and computed tomographic (CT) images characteristics in the patients with COVID-19 infection: What should radiologists need to know?. Journal of X-ray science and technology 2020; 28(3):369-381. doi: 10.3233/XST-200670.

18. Wang H, Wei R, Rao G, Zhu J, et al. Characteristic CT findings distinguishing 2019 novel coronavirus disease (COVID-19) from influenza pneumonia. $\mathrm{Eu}$ ropean Radiology 2020; 30(9):4910-4917. doi: 10.1007/s00330-020-06880-z

19. Wong HY, Lam HY, Fong AH, Leung ST, et al. Frequency and distribution of chest radiographic findings in COVID-19 positive patients. Radiology. 2020; 296(2):E72-E78. doi: 10.1148/radiol.2020201160

20. Xie X, Zhong Z, Zhao W, Zheng C, et al. Chest CT for typical 2019-nCoV pneumonia: relationship to negative RT-PCR testing. Radiology. 2020; 200343. DOI: $10.1148 /$ radiol.2020200343 